\title{
Absorption Enhancement in 2D Nanocrystal Superlattices through Near-Field Dipolar Coupling: A Novel Optical Phenomenon at the Nanoscale
}

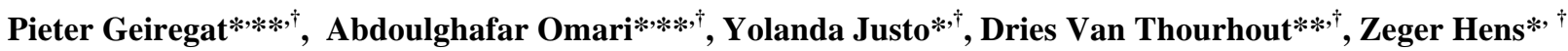 \\ **Photonics Research Group, INTEC-IMEC, University of Ghent, Ghent (Belgium) \\ $\dagger$ Center for Nano- and Biophotonics, University of Ghent, Belgium \\ *Physics and Chemistry of Nanostructures Group, University of Ghent, Ghent (Belgium) \\ Authore-mail address: Pieter.Geiregat@Ugent.be
}

\begin{abstract}
We demonstrate giant and broadband enhancement of the nanocrystal absorption cross section in close packed nanocrystal superlattices, which is the first report on a collective optical phenomenon in this type of self-assembled metamaterials to date.
\end{abstract}

Colloidal nanocrystals (NC) are nanometer-sized semiconductor crystals obtained through a solution-based chemical synthesis. Apart from changing their composition, the optical properties (such as the absorption cross section and band gap) of the crystals can be tuned by varying their size, a direct result of strong quantum confinement in these structures. Moreover, their easy deposition allows for cheap bottom-up fabrication. For these reasons, nanocrystals are thought to be viable candidates for opto-electronic applications such as the fabrication of solar cells, light emitting diodes, photo-detectors, etc.... In such a device context, nanocrystals will very often be deposited in thin layers. However, as NCs are obtained through wet chemical synthesis, their optical properties (e.g. absorption cross section) are typically evaluated in solution using effective medium approaches, which assume no electromagnetic coupling between the absorbing dipoles. It is questionable that this assumption still holds for a close packed film of nanocrystals where those dipoles tend to couple through electromagnetic multipolar interactions. Using properties measured in solution to evaluate and study the performance and physics of thin film devices would therefore be incorrect, or at least a serious simplification. The basic idea of our approach to understand such close-packed dipoles is that of the 'coupled dipole model' (CD): the internal field of a particle in close proximity to other dipoles will be a superposition of the influence of the external field and the induced dipolar fields of the neighboring particles (see Figure 1, right). To quantify this idea, we will focus on the NC absorption cross section and we define an 'enhancement factor ' $\mathrm{E}$ ', being the ratio between the absorption cross section in film to the cross section in solution and try to obtain E both through theory (via the coupled dipole model) and experiment ${ }^{2}$.

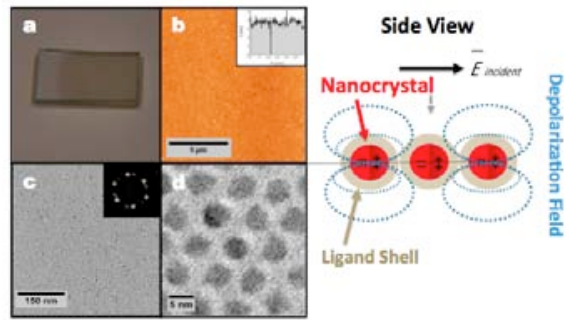

Figure 1: Close-up of nanocrystal monolayer (a: Image of monolayer on glass, b: AFM surface morphology, c: TEM image and d: TEM close-up showing local hexagonal order) and schematic of near-field coupling mechanism proposed in this work.

To access the enhancement E experimentally, we measure the absorption spectrum of single and multiple close packed monolayers of nanocrystals on glass substrates (see Figure 1) using a standard UV-VIS-NIR spectrophotometer. The layers (essentially a meta-material) are deposited using Langmuir-Blodgett deposition ${ }^{1}$ and are well defined (i.e. particle density and lattice symmetry are uniform over large areas). The enhancement predicted by our $\mathrm{CD}$ interpretation is substantial, leading to a size-dependent value of $\mathrm{E}$ up to 5 and 4 for $\mathrm{PbS}$ and $\mathrm{CdSe}$ monolayers respectively (see figure 2, left). To test our CD modeling, we use 2D single component monolayer as a reference system. We vary the parameters experimentally accessible to us: particle composition and size (i.e. $\mathrm{PbS}$ and $\mathrm{CdSe}$ ), inter-particle spacing (through ligand spacer length) and dipole strength (through $\mathrm{PbS} / \mathrm{CdS}$ core-shell particles at fixed inter-distance). The results are shown in Figures $2 \& 3$ and agree very well with theoretical predictions based on the CD model which confirms our idea that near-field electrostatic interactions are present in these NC superlattices, a phenomenon never observed before in these nanoscale metamaterials. 

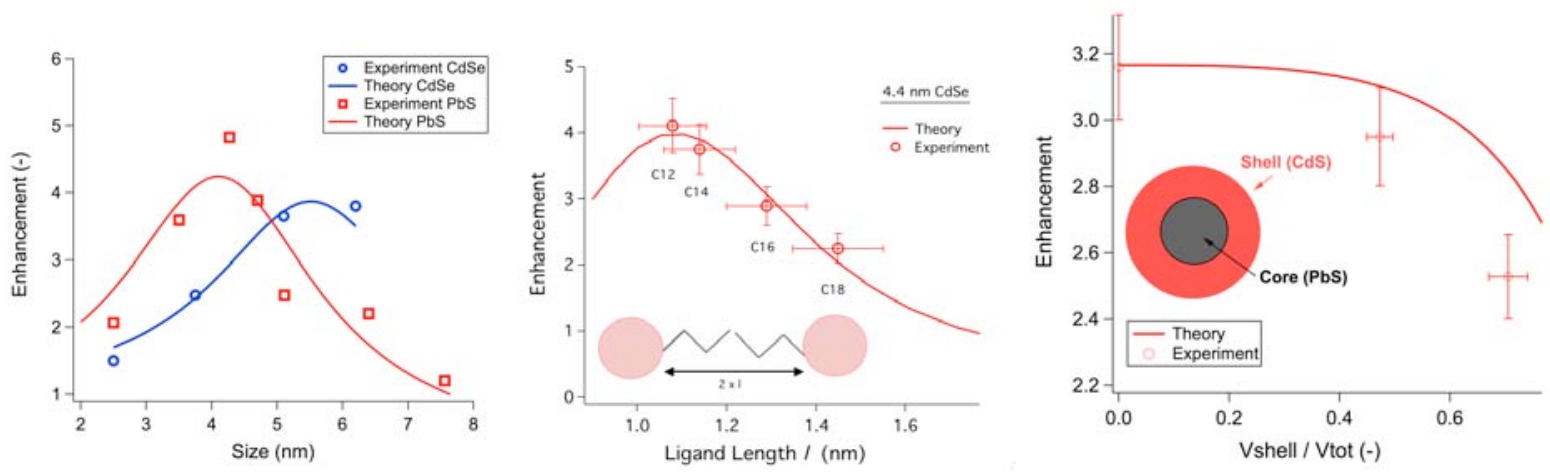

Figure 2: (left) Absorption cross section enhancement $\mathrm{E}$ at $400 \mathrm{~nm}$ and $355 \mathrm{~nm}$ for PbS and CdSe respectively, (middle) enhancement for $4.4 \mathrm{~nm}$ $\mathrm{CdSe}$ as function of ligand spacing 1 and (right) enhancement for $\mathrm{PbS} / \mathrm{CdS}$ core/shell nanocrystals for varying core to shell ratio.

Whether this new phenomenon can play a role of interest in real devices, depends on two additional issues: Is the enhancement broadband and does it extend beyond the monolayer thickness? As is evident in Figure 3 (left) for a $\mathrm{PbS}$ nanocrystal, the enhancement is indeed broadband and extends from the UV down to the band gap of the nanocrystal in the near-infrared. We can understand this based on the non-resonant polarizability of the individual semiconducting crystals, opposed to e.g. metal nanocrystals showing distinct multipolar resonances.
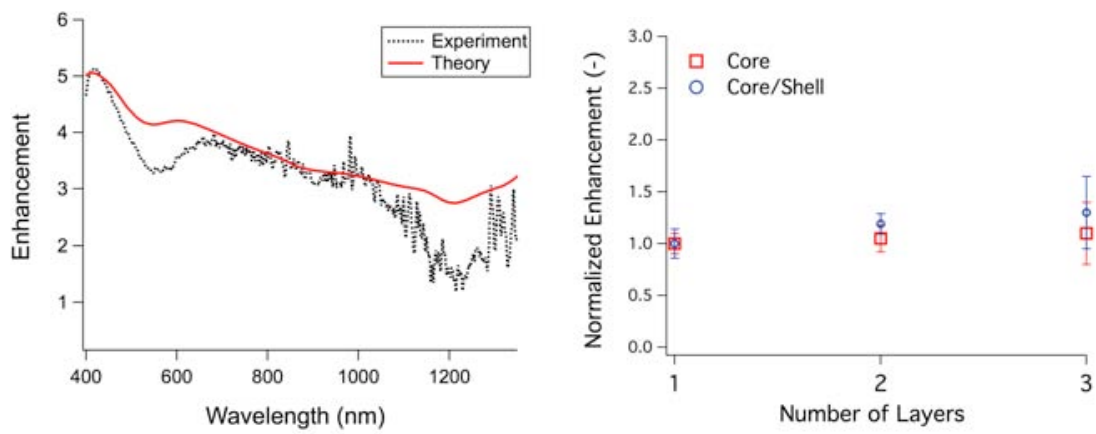

Figure 3 : (left) Broadband nature of the absorption enhancement for $4.3 \mathrm{~nm} \mathrm{PbS}$ and (right) scaling of enhancement

The amount of material needed to absorb a given fraction of incident light competes with the extraction of photogenerated carriers: thicker cells absorb more light, but do not allow for efficient carrier extraction and vice versa. Our absorption enhancement mechanism could offer part of the solution since it would allow fabrication of thinner cells with light absorption equal to that of non-optimized thicker cells. Our model can be extended to analyze these technologically relevant multilayers. It turns out the enhancement is also present in these stacked systems in an additive way (see Figure 3, right): Dipole coupling occurs between particles in the same layer, but does not occur between different layers in the stack. This allows us to stack the layers until a certain desired absorption is achieved, without losing the effect of absorption enhancement.

We demonstrated and modeled for the first time a new and exciting collective optical effect in self-assembled nanocrystal superlattices. The (experimentally validated) model presented here provides a general framework to understand the observed intricate optical properties and presents new questions regarding the difference between sand p-polarized light and the impact optimal coupling on the radiative lifetime. Moreover, it gives a basis to understand the optical properties of more complex, possibly hybrid superlattices, for example, containing nanocrystals of different sizes or different materials allowing for more advanced device functionalities.

\section{References}

1. Justo, Y., Moreels, I., Lambert, K. \& Hens, Z. Langmuir-Blodgett monolayers of colloidal lead chalcogenide quantum dots: morphology and photoluminescence. Nanotechnology 21, 295606 (2010).

2. Geiregat, P., Justo, Y., Abé, S., Flamee, S. \& Hens, Z. Giant and Broadband Absorption Enhancement in Colloidal Quantum Dot Monolayers through Dipolar Coupling. ACS Nano (2013).doi:10.1021/nn305524a 